Artículo científico

Volumen 30(3):647-658. Septiembre-diciembre, 2019 e-ISSN 2215-3608, doi:10.15517/am.v30i3.36327 http://www.revistas.ucr.ac.cr/index.php/agromeso

\title{
Beauveria bassiana con flupyradifurone para controlar Trialeurodes vaporariorum Vuill en el cultivo del ejote francés ${ }^{1}$
}

\section{Beauveria bassiana with flupyradifurone for the control of Trialeurodes vaporariorum Vuill in French beans crop}

\author{
Claudia Elizabeth Toledo-Perdomo²
}

1 Recepción: 25 de febrero, 2019. Aceptación: 10 de junio, 2019. Este trabajo formó parte del proyecto de Investigación "Evaluación del sinergismo del hongo entomopatógeno Beauveria bassiana Vuill con plaguicidas químicos flupyradifurone para el control de mosca blanca (Insecta: Hemiptera) en el cultivo del ejote francés en el departamento de Chimaltenango", financiado por la Dirección General de Investigación de la Universidad de San Carlos de Guatemala con partida presupuestal 4.8.57.246.

2 Universidad de San Carlos de Guatemala, Centro Universitario de Santa Rosa, Cuilapa, Santa Rosa, Guatemala. toledo.perdomo@gmail.com (https://orcid.org/0000-0003-2281-3216).

\section{Resumen}

Introducción. Trialeurodes vaporariorum (Westwood) es una de las principales plagas del ejote francés (Phaseolus vulgaris L.), leguminosa para consumo en vainas inmaduras, y es vector del virus del mosaico dorado, que afecta la calidad y rendimiento del cultivo, con pérdidas hasta del 100\%. Su control es poco efectivo, debido a que muchos productos químicos ya no controlan la plaga. El ejote francés dentro de los productos no tradicionales en Guatemala, ocupa el segundo lugar en la exportación. Asegurar un buen manejo fitosanitario con baja carga química por plaguicidas, son factores importantes para poder exportarlo. Objetivo. La investigación tuvo por objetivo determinar la efectividad de la combinación entre flupyradifurone y Beauveria bassiana para el control de la mosca blanca en el cultivo del ejote francés. Materiales y métodos. El estudio se realizó de febrero a diciembre de 2018 en Parramos, Chimaltenango, Guatemala. Se establecieron siete parcelas de $100 \mathrm{~m}^{2}$, correspondientes a cada tratamiento. Se muestrearon moscas blancas semanalmente durante dos ciclos de cultivo, también se colectaron en cada parcela enemigos naturales de estas. Se realizaron tres aplicaciones de cada tratamiento en cada ciclo del cultivo y se muestrearon las plantas divididas en tres estratos (alto, medio, bajo). Resultados. Los tratamientos que presentaron el mejor control de la población de mosca blanca (ninfas y adultos) al realizar la prueba de Tukey $(0,05)$, fueron flupyradifurone $\left(11 \mathrm{ha}^{-1}\right)+B$. bassiana y flupyradifurone $\left(0,751 \mathrm{ha}^{-1}\right)+B$. bassiana. La mayor distribución de las poblaciones de mosca blanca se encontró en los estratos alto y medio. Conclusión. La combinación de flupyradifurone $\left(0,751 \mathrm{ha}^{-1}\right)$ con $B$. bassiana puede ser empleado dentro de un manejo integrado de plagas, y se presenta como una alternativa de control de la mosca blanca, que ha adquirido resistencia a algunos agroquímicos.

Palabras clave: Trialeurodes vaporariorum, gestión de lucha integrada, Phaseolus vulgaris, estratos.
Abstract
Introduction. Trialeurodes vaporariorum (Westwood) is one of the main pests of the French bean (Phaseolus vulgaris L.), legume for consumption in inmature pods, and is a vector of the golden mosaic virus, which affects 
the quality and yield of the crop reaching losses of up to $100 \%$, being difficult to control because many chemicals no longer control the pest. The French bean within the non-traditional products in Guatemala, occupies the second place in the export. Ensuring a good phytosanitary management with low chemical load by pesticides are important factors to be able to export it. Objective. The objective of the investigation was to determine the effectiveness of the combination between Flupyradifurone and Beauveria bassiana for the control of whitefly in French bean cultivation. Materials and methods. The study was conducted from February to December 2018 in Parramos, Chimaltenango, Guatemala. Seven plots of $100 \mathrm{~m}^{2}$ were established corresponding to each treatment. Weekly, whiteflies were sampled during two cultivation cycles, and natural enemies of these were also collected on each plot. Three applications of each treatment were made in each crop cycle, and the plants were sampled divided into three strata (high, medium, low). Results. The treatments that showed the best control of the whitefly population (nymphs and adults) when performing the Tukey test $(0,05)$, were flupyradifurone $\left(11 \mathrm{ha}^{-1}\right)+B$. bassiana, and flupyradifurone $\left(0.751 \mathrm{ha}^{-1}\right)+B$. bassiana. The largest distribution of whitefly populations was found in the upper and middle strata. Conclusion. The combination of flupyradifurone $(0,05)$ with $B$. bassiana can be used within an integrated pest management, and is presented as an alternative control of whiteflies, which has acquired resistance to some agrochemicals.

Keywords: Trialeurodes vaporariorum, integrated pest management, Phaseolus vulgaris, strata.

\section{Introducción}

El cultivo de las arvejas y del ejote francés (Phaseolus vulgaris L.), leguminosa para consumo en vainas inmaduras, ocupa los primeros lugares de exportación dentro del sector de vegetales no tradicionales en Guatemala, donde, se ha exportado aproximadamente 21000 t de ejote francés, favoreciendo al sector agrícola y rural, principalmente en el altiplano de Guatemala, Jalapa, Alta Verapaz y Baja Verapaz (AGEXPORT, 2016). En el año 2013 el ejote francés generó 2549828 jornales y 9444 empleos permanentes en Guatemala, y para el 2014 el país exportó 1667 tm de la cosecha del cultivo (Contreras, 2014).

Para poder exportar la cosecha del ejote francés se debe cumplir con una serie de requisitos de los mercados de destino, tales como las normas de calidad de la cosecha y el cumplimiento de los límites máximos de residuos (MRL) de plaguicidas. Por ejemplo, para Estados Unidos el MRL de flupyradifurones es $0,2 \mathrm{ppm}$ y el de $B$. bassiana está exento; mientras que para la Unión Europea el MRL de flupyradifurone es de 0,01 ppm y el de $B$. bassiana está exento; en este último caso, los MRL están establecidos en los reglamentos UE 1107 de 2009 y UE 396 de 2005 (EC, 2017; AGEXPORT, 2018; U.S. Government Publishing Office, 2019).

Los principales destinos de exportación del ejote francés son Estados Unidos y Canadá con el $94 \%$ de las exportaciones, y el $6 \%$ restante al mercado europeo (Cámara del Agro y Agrequima, 2015). La calidad del ejote para exportación también está vinculada a características físicas tales como la forma, el color, ausencia de manchas o daños en la vaina.

Las especies de mosca blanca (Bemisia tabaci) (Gennadius, 1889) y Trialeurodes vaporarorium (Westwood, 1856) son plagas importantes en diversos cultivos, las cuales están ampliamente distribuidas en regiones tropicales y subtropicales del mundo, donde afectan más de 600 especies de plantas cultivadas y silvestres. En su alimentación, la mosca blanca provoca un debilitamiento en la planta por la extracción de nutrientes y la transmisión de virus. En el ejote francés estos insectos son transmisores de los virus del mosaico dorado y el mosaico dorado amarillo, los cuales causan pérdidas en rendimiento de hasta el 100 \% (Morales y Anderson, 2001; Jones, 2003; Villar-Sánchez et al., 2003; Cuellar y Morales, 2006; Morales, 2006).

Tradicionalmente el control de la mosca blanca ha sido con productos químicos, de los cuales se tiende a abusar y/o a emplear malas prácticas, por ello, se ha observado la resistencia a varios grupos de insecticidas, provocando 
un incremento en las poblaciones del insecto en los campos, haciendo más difícil el control de la plaga (Kandil et al., 2008; Campuzano-Martínez et al., 2010; Macías-Flores et al., 2013; Naveen et al., 2017).

Al evaluar los insecticidas cyazypyr, flupyradifurone, pyrifluquinazol, sulfoxaflor, zeta-cipermetrina y befentrina para el control de huevos y ninfas de la mosca blanca (Bemisia tabaci), Smith y Giurcanu (2013) determinaron que cyazypyr, flupyradifurone, pyrifluquinazol y sulfoxaflor presentaron mejor control sobre huevos y ninfas, que los insecticidas zeta-cipermetrina y befentrina.

Cuando se comparó la eficiencia de flupyradifurone con thiametoxam para el control del virus de rizado amarillo de la hoja de tomate (TYLCV), transmitido por la mosca blanca, se determinó que flupyradifurone suprimió en un $85 \%$ el virus, mientras que, thiametoxam en un $25 \%$ (Roditakis et al., 2017).

Flupyradifurone ha estado en el mercado desde el año 2015 en los Estados Unidos, donde las evaluaciones han demostrado su efecto en plagas succionadoras, especialmente para el control de áfidos y mosca blanca, plagas que han demostrado tener mecanismos metabólicos de resistencia a insecticidas neonicotinoides (Nauen et al., 2015).

De los entomopatógenos Beauveria bassiana, Paecilomyces sp. y Lecanicillium lecanii, utilizados bajo condiciones de laboratorio para el control de ninfas de mosca blanca en foliolos de fríjol, el más eficiente fue $B$. bassiana con un 96,5\%, seguido de Paecilomyces sp. con un 81,8 \% y L. lecanii, con el $70 \%$ (Espinel et al., 2008).

Estudios realizados sobre la integración de un plaguicida sintético con un entomopatógeno (Metarrhizium anisopliae), determinaron que esta combinación mostró mayor efecto en el manejo de la población que al ser utilizados individualmente, reduciendo los costos de producción y residuos químicos en los productos (Ouma et al., 2014). El objetivo de esta investigación fue determinar la efectividad de la combinación entre flupyradifurone y Beauveria bassiana para el control de la mosca blanca en el cultivo del ejote francés.

\section{Materiales y métodos}

\section{Localización}

En el periodo comprendido entre febrero y diciembre de 2018, se establecieron siete parcelas del ejote francés (Phaseolus vulgaris L.) variedad Serenguetti (Syngenta, 2019). Estas fueron seleccionadas en plantaciones comerciales de ejote francés para la exportación, correspondientes a cada uno de los tratamientos evaluados.

Las parcelas estaban localizadas en el departamento de Chimaltenango, municipio de Parramos, Guatemala; ubicado a una altitud de 1760 msnm (AMSA, 2005; INE, 2013), coordenadas 14.592171, -90.809229.

\section{Recolección de datos}

Se evaluaron en cada parcela cinco plantas al azar, cada una se dividió en tres estratos: alto, medio y bajo (dos hojas por estrato, evaluando un total de seis hojas) y, dos ciclos del cultivo. Los muestreos se realizaron un día antes y un día después de la primera aplicación, posteriormente, una vez por semana, haciendo un total de seis muestreos por ciclo de cultivo.

Se calculó el tamaño de la muestra, con los siguientes criterios estadísticos:

Se tomaron treinta muestras por tratamiento, que corresponde a seis hojas colectadas en cinco plantas, con un nivel de significancia de 0,05 , y un poder estadístico del $80 \%(\beta=0,20)$. El efecto esperado fue igual a la desviación estándar de las respuestas. La respuesta esperada fue el número de individuos de mosca blanca por cada uno de los siete tratamientos. La comparación de las poblaciones después de aplicados los tratamientos sirvieron para evaluar la efectividad de cada uno de ellos. 
En los conteos en campo se tomaron en cuenta el número de moscas blancas adultas y el número de ninfas encontradas en las hojas que se seleccionaron en el muestreo. Las muestras se seleccionaron de acuerdo con una adaptación de las metodologías sugeridas para el estudio de las poblaciones de mosca blanca de Bueno et al. (2005) y Bernal et al. (2008). Se contaron los individuos presentes en el haz y en el envés de las hojas, para este último, se tomó cuidadosamente y muy lentamente el extremo apical de cada hoja y se levantó para tener visibilidad y evitar el vuelo de las moscas.

\section{Tratamientos, técnicas e instrumentos}

Los tratamientos que se evaluaron fueron: flupyradifurone $\left(0,751 \mathrm{ha}^{-1}\right)$, flupyradifurone $\left(11 \mathrm{ha}^{-1}\right)$, flupyradifurone $\left(0,751 \mathrm{ha}^{-1}\right)+$ Beauveria bassiana $\left(4,541 \mathrm{ha}^{-1}\right)$, flupyradifurone $\left(11 \mathrm{ha}^{-1}\right)+$ Beauveria bassiana $\left(4,541 \mathrm{ha}^{-1}\right)$, Beauveria bassiana $\left(4,541 \mathrm{ha}^{-1}\right)$, control comercial, como control absoluto, y un control negativo (agua).

El control comercial consistió en tres aplicaciones de lambada cyhalothrin, el cual es comúnmente utilizado por los productores para el control de la mosca blanca.

Las dosis de flupyradifurone de acuerdo con las recomendaciones para Guatemala de la casa comercial Sivanto Prime 20 SL.

La primera aplicación se realizó cuando la planta tenía quince días después de haber germinado. Para obtener una buena aplicación de los tratamientos, se realizó la calibración del equipo, y así, garantizarel suministro de la dosis calculada y cobertura total del área foliar deseada. Se tomaron en cuenta el volumen calibrado y la descarga, y se calculó la cantidad de sustancia de prueba que se necesitó agregar a la mezcla; este procedimiento se realizó tres veces. Se tomó en cuenta si la variación entre calibraciones era mayor al $5 \%$, se continuó hasta obtener tres calibraciones consecutivas que estuvieran dentro del rango establecido (no mayor al $5 \%$ ).

Luego de la primera calibración, solo se necesitó una verificación, se consideró que si la misma se encontraba dentro del $5 \%$ respecto a la calibración original, se tomaba como válido; si estaba fuera del rango, fue necesario realizar una calibración completa. Finalmente, se realizó el cálculo la eficiencia de la aplicación y, este dato, se transformó en 1 ha $^{-1}$ y se comparó con el volumen establecido para cada parcela. Al total del producto cosechado de cada tratamiento, para clasificarlo para la exportación, se le evaluaron características como el tamaño del ejote, forma, grosor y color; también se tomó en cuenta el daño causado por virosis (vainas con manchas cloróticas, malformación, tamaño pequeño), y se eliminaron aquellas plantas con una afectación avanzada. Los datos obtenidos se expresaron en porcentaje. Entre tratamientos, se comparó el rendimiento en gramos de ejote con calidad para exportación cosechado por parcela.

Para la determinación de las especies de mosca blanca que fueron colectadas se emplearon las claves taxonómicas de Caballero (1992) y Carapia y Castillo (2013), y los descriptores de las especies de mosca blanca de Salas y Mendoza (1995).

\section{Procesamiento y análisis de los datos}

Se analizaron las poblaciones de la mosca blanca durante el tiempo de las aplicaciones de los tratamientos, considerando los estratos establecidos. El análisis fue descriptivo para cada tratamiento y cada estrato; el procesamiento de datos se realizó con el programa Statistica.

A los resultados obtenidos de cada uno de los tratamientos se les realizó una prueba de normalidad, debido a que su comportamiento no fue normal, fue necesaria la transformación de los resultados a logaritmo natural (Ln) de adultos y ninfas. Con los datos transformados se realizó un análisis de residuos, donde se comprobó que el 
modelo bivariado fuera adecuado para las variables, debido a que estas se distribuyeron aleatoriamente alrededor del modelo en forma normal.

Se realizó un análisis de varianza del porcentaje de la reducción de las poblaciones de mosca blanca por los tratamientos evaluados, con un nivel de significancia de $0,05 \%$; este análisis fue de tres vías (población de adultos, población de ninfas y población total), con medias repetidas para determinar las diferencias entre tratamientos, controlando diferencias posibles dentro de los estratos de la planta y el ciclo del cultivo.

Se realizó la prueba de Tukey con un nivel de significancia de $0,05 \%$ para conocer las diferencias entre los tratamientos.

\section{Resultados}

\section{Evaluación de los tratamientos}

El análisis de varianza y del total de la población (adultos y ninfas) de cada uno de los tratamientos, con la prueba de Tukey (Cuadro 1 y 2), mostró diferencias significativas cuando se utilizó flupyradifurone $\left(11\right.$ ha $\left.^{-1}\right)+B$. bassiana, estos presentaron un mejor control de la población en comparación con los demás tratamientos evaluados.

El tratamiento flupyradifurone $\left(11 \mathrm{ha}^{-1}\right)+B$. bassiana, fue el que realizó el mejor control de las poblaciones de mosca blanca para todas las categorías de Ln, y mostró diferencias significativas con los demás tratamientos, excepto con B. bassiana + flupyradifurone $\left(0,75 \mathrm{l} \mathrm{ha}^{-1}\right)$. Este tratamiento también fue el mejor significativamente para el control de Ln ninfas y Ln adultos (Cuadro 2).

El mejor rendimiento del cultivo en las parcelas lo presentaron aquellos en que se aplicó flupyradifurone $\left(11 \mathrm{ha}^{-1}\right)+B$. bassiana y flupyradifurone $\left(0,751 \mathrm{ha}^{-1}\right)+B$. bassiana; seguido de los tratamientos $B$. bassiana, flupyradifurone $\left(11 \mathrm{ha}^{-1}\right)$ y flupyradifurone $\left(0,751 \mathrm{ha}^{-1}\right)$; de último, con los rendimientos más bajos se observaron los testigos con agua y absoluto (Cuadro 3 ).

Cuadro 1. Análisis de varianza con datos sin transformar y transformados a Ln (logaritmo natural) de la población (adultos, ninfas y total de las poblaciones) en los tratamientos flupyradifurone y Beauveria bassiana para el control de la mosca blanca en el cultivo del ejote francés (Phaseolus vulgaris var. Serenguetti). Chimaltenango, Guatemala. 2018.

Table 1. Analysis of variance with untransformed data and transformed to Ln (natural logarithm) of the population (adults, nymphs and total populations) in the Flupyradifurone and Beauveria bassiana treatments for the control of the whitefly in the crop french beans (Phaseolus vulgaris var. Serenguetti). Chimaltenango, Guatemala. 2018.

\begin{tabular}{lcccccccccc}
\hline $\begin{array}{l}\text { Datos sin } \\
\text { transformar } \\
\text { Variable }\end{array}$ & $\mathbf{N}$ & $\mathbf{N} *$ & Media & Desv. Est. & Mínimo & Q1 & Mediana & Q3 & Máximo & Asimetría \\
\hline Adultos & 315 & 0 & 41,53 & 54,25 & 0,00 & 6,00 & 17,00 & 60,00 & 352,00 & 2,39 \\
Ninfas & 315 & 0 & 23,97 & 32,85 & 0,00 & 2,00 & 13,00 & 32,00 & 225,00 & 2,73 \\
Total & 315 & 0 & 65,50 & 74,69 & 0,00 & 15,00 & 40,00 & 83,00 & 417,00 & 2,01 \\
\hline $\begin{array}{l}\text { Datos } \\
\text { transformados }\end{array}$ & & & & & & & & & & \\
Variable & $\mathbf{N}$ & $\mathbf{N} *$ & Media & Desv. Est. & Mínimo & $\mathbf{Q 1}$ & Mediana & $\mathbf{Q 3}$ & Máximo & Asimetría \\
\hline Ln adultos & 290 & 25 & 3,0873 & 1,2905 & 0,00 & 2,0794 & 3,0678 & 4,1471 & 5,8636 & $-0,10$ \\
Ln ninfas & 254 & 61 & 2,8019 & 1,1951 & 0,00 & 2,1972 & 2,8904 & 3,6441 & 5,4161 & $-0,48$ \\
Ln total & 293 & 22 & 3,6989 & 1,1519 & 0,00 & 2,9444 & 3,7612 & 4,5109 & 6,0331 & $-0,43$ \\
\hline
\end{tabular}


Cuadro 2. Prueba de Tukey para Ln adultos, Ln ninfas y Ln total de la población de mosca blanca en el cultivo del ejote francés (Phaseolus vulgaris var. Serenguetti) por tratamiento: flupyradifurone y Beauveria bassiana. Chimaltenango, Guatemala. 2018.

Table 2. Tukey test for Ln adults, Ln nymphs and total Ln of the whitefly population in the French bean (Phaseolus vulgaris var. Serenguetti) culture by treatment: Flupyradifurone and Beauveria bassiana. Chimaltenango, Guatemala. 2018.

\begin{tabular}{|c|c|c|c|c|c|c|}
\hline \multicolumn{7}{|c|}{ Ln total (adultos y ninfas) } \\
\hline Tratamiento & $\mathbf{N}$ & Media & & upa & ión & \\
\hline Testigo + agua & 43 & 4,40772 & $\mathrm{~A}$ & & & \\
\hline Testigo absoluto & 43 & 4,23758 & A & & & \\
\hline Flupyradifurone $\left(0,751 \mathrm{ha}^{-1}\right)$ & 42 & 3,67001 & & B & & \\
\hline Beauveria bassiana & 43 & 3,58234 & & $\mathrm{~B}$ & $\mathrm{C}$ & \\
\hline Flupyradifurone $\left(11 \mathrm{ha}^{-1}\right)$ & 42 & 3,56662 & & $\mathrm{~B}$ & $\mathrm{C}$ & \\
\hline B. bassiana + flupyradifurone $\left(0,751 \mathrm{ha}^{-1}\right)$ & 40 & 3,18347 & & & $\mathrm{C}$ & $\mathrm{D}$ \\
\hline B. bassiana + flupyradifurone $\left(11 \mathrm{ha}^{-1}\right)$ & 40 & 2,82558 & & & & $\mathrm{D}$ \\
\hline \multicolumn{7}{|c|}{ Ln adultos } \\
\hline Testigo + agua & 42 & 3,59565 & A & & & \\
\hline Testigo absoluto & 43 & 3,36345 & A & $\mathrm{B}$ & & \\
\hline Flupyradifurone $\left(0,751 \mathrm{ha}^{-1}\right)$ & 42 & 3,00776 & & B & $\mathrm{C}$ & \\
\hline Beauveria bassiana & 42 & 2,97952 & & & $\mathrm{C}$ & \\
\hline Flupyradifurone $\left(11 \mathrm{ha}^{-1}\right)$ & 42 & 2,80208 & & & $\mathrm{C}$ & \\
\hline B. bassiana + flupyradifurone $\left(0,751 \mathrm{ha}^{-1}\right)$ & 40 & 2,65851 & & & $\mathrm{C}$ & $\mathrm{D}$ \\
\hline B. bassiana + flupyradifurone $\left(11 \mathrm{ha}^{-1}\right)$ & 39 & 2,40791 & & & & $\mathrm{D}$ \\
\hline \multicolumn{7}{|c|}{ Ln ninfas } \\
\hline Testigo + agua & 41 & 3,39928 & A & & & \\
\hline Testigo absoluto & 39 & 3,27364 & $\mathrm{~A}$ & & & \\
\hline Beauveria bassiana & 35 & 2,69946 & & B & & \\
\hline Flupyradifurone $\left(11\right.$ ha $\left.^{-1}\right)$ & 38 & 2,62489 & & B & & \\
\hline Flupyradifurone $\left(0,751 \mathrm{ha}^{-1}\right)$ & 38 & 2,60363 & & B & & \\
\hline B. bassiana + flupyradifurone $\left(0,751 \mathrm{ha}^{-1}\right)$ & 33 & 2,23765 & & B & $\mathrm{C}$ & \\
\hline B. bassiana + flupyradifurone $\left(11 \mathrm{ha}^{-1}\right)$ & 30 & 1,95738 & & & $\mathrm{C}$ & \\
\hline
\end{tabular}

Nivel de significancia de 0,05. Las medias que no comparten una letra son significativamente diferentes / Significance level of 0.05 . Means that do not share a letter are significantly different.

\section{Distribución de la población y especies de mosca blanca}

La distribución poblacional de adultos de mosca blanca dentro de la planta, mostró diferencias significativas entre los tres estratos (Cuadros 4 y 5).

El estudio taxonómico de los especímenes de mosca blanca colectados mostró la existencia de dos especies: Bemisia tabaci (Gennadius) y Trialeurodes vaporariorum (Westwood), de las cuales la más abundante fue la segunda con un porcentaje del $95 \%$ del total de las moscas blancas colectadas. 
Cuadro 3. Cosecha en gramos de los dos ciclos del cultivo evaluados en campo de cada tratamiento con flupyradifurone y Beauveria bassiana, en ejote francés (Phaseolus vulgaris var. Serenguetti) para el control de la mosca blanca. Chimaltenango, Guatemala. 2018. Table 3. Harvest in grams of the two crop cycles evaluated in the field of each treatment with flupyradifurone and Beauveria bassiana, in French bean (Phaseolus vulgaris var. Serenguetti) for whitefly control. Chimaltenango, Guatemala. 2018.

\begin{tabular}{|c|c|c|c|c|c|c|c|c|}
\hline $\begin{array}{l}\text { Ciclo del } \\
\text { cultivo }\end{array}$ & Peso (g) & $\begin{array}{c}\text { flupyradi- } \\
\text { furone }(1 \text { l } \\
\left.\text { ha' }^{-1}\right)\end{array}$ & $\begin{array}{c}\text { flupyra- } \\
\text { difurone } \\
\left(0,75 \text { l ha-1 }^{-1}\right)\end{array}$ & $\begin{array}{c}\text { B. bassiana } \\
+ \text { flupyra- } \\
\text { difurone }(1 \\
\left.\text { l ha }^{-1}\right)\end{array}$ & $\begin{array}{c}\text { B. bassiana } \\
\text { + flupyra- } \\
\text { difurone } \\
\left(0,75 \text { l ha }^{-1}\right)\end{array}$ & B. bassina & $\begin{array}{c}\text { Control } \\
\text { agua }\end{array}$ & $\begin{array}{c}\text { Control } \\
\text { total }\end{array}$ \\
\hline \multirow[t]{3}{*}{1} & Neto* & 47954 & 47917 & 48995 & 48512 & 47938 & 46236 & 46321 \\
\hline & Rechazo** & 527 & 508 & 128 & 132 & 219 & 874 & 796 \\
\hline & Total $* * *$ & 47427 & 47409 & 48384 & 48380 & 47719 & 45362 & 45525 \\
\hline \multirow[t]{3}{*}{2} & Neto* & 48927 & 48846 & 50122 & 50108 & 48834 & 47013 & 46998 \\
\hline & Rechazo** & 658 & 669 & 204 & 231 & 335 & 897 & 911 \\
\hline & Total $* * *$ & 48269 & 48177 & 49918 & 49877 & 48499 & 46116 & 46087 \\
\hline
\end{tabular}

*Neto: cosecha de cada parcela correspondiente a cada tratamiento con características para la exportación. ** Rechazo: del total cosechado se revisó descartando el ejote que no cumple con las características para la exportación. ***Total: resta el rechazo al neto / *Net: harvest of each plot corresponding to each treatment with characteristics for export. ** Rejection: of the total harvested was reviewed discarding the french bean that does not comply with the characteristics for export. *** Total: subtract the rejection from the net.

Cuadro 4. Comparaciones para Ln adultos por parejas del método LSD Fisher por estrato de la planta del ejote francés (Phaseolus vulgaris var. Serenguetti). Chimaltenango, Guatemala, 2018.

Table 4. Comparisons for Ln adults by pairs of the Fisher LSD method by stratum of the French bean plant (Phaseolus vulgaris var. Serenguetti). Chimaltenango, Guatemala, 2018.

\begin{tabular}{lccccc}
\hline Época & Estrato & N & Media & Agrupación \\
\hline Seca & estrato alto & 56 & 3,99333 & A & B \\
& estrato medio & 49 & 3,63209 & 2,02568 & C \\
\hline Lluviosa & estrato bajo & 47 & 4,16093 & A & B \\
& estrato alto & 49 & 2,79948 & 1,37764 & C \\
& estrato medio & 49 & 40 & & \\
\hline
\end{tabular}

Nivel de significancia de 0,05. Las medias que no comparten una letra son significativamente diferentes / Significance level of 0.05 . Means that do not share a letter are significantly different.

\section{Discusión}

Los resultados obtenidos (Cuadros 1 y 2) indicaron que los tratamientos que consistieron en la combinación de flypyradifurone con el hongo entomopatógeno B. bassiana, pueden ser una valiosa herramienta para considerarlos dentro de un programa de manejo integrado de plagas para el control de la mosca blanca en el ejote francés, con el fin de reducir el uso de productos como tiametoxam e imidacloprid, ambos neonicotinoides, los cuales se utilizan regularmente en el control de la mosca blanca y son dañinos para las abejas. Estudios realizados en estos 
Toledo-Perdomo: Beauveria bassiana con flupyradifurone para controlar mosca blanca

Cuadro 5. Estadísticos descriptivos para Ln de adultos por estrato de la planta de ejote francés (Phaseolus vulgaris var. Serenguetti), en época seca y lluviosa. Chimaltenango, Guatemala. 2018.

Table 5. Descriptive statistics for Ln of adults by stratum of the French bean plant (Phaseolus vulgaris var. Serenguetti), in dry and rainy season. Chimaltenango, Guatemala. 2018.

\begin{tabular}{lccccccc}
\hline Variable & Estrato & $\mathbf{N}$ & $\mathbf{N}^{*}$ & Media & DE & Mediana & Conteo medio \\
\hline Ln adultos & estrato alto & 56 & 0 & 3,868 & 1,078 & 4,103 & 48 \\
Época & estrato bajo & 47 & 9 & 2,127 & 0,876 & 2,303 & 8 \\
seca & estrato medio & 49 & 7 & 3,698 & 1,080 & 3,871 & 40 \\
\hline Ln adultos & estrato alto & 49 & 0 & 4,1590 & 0,5824 & 4,0254 & 64 \\
Época & estrato bajo & 40 & 9 & 1,4159 & 0,4847 & 1,3863 & 4 \\
lluviosa & estrato medio & 49 & 0 & 2,798 & 0,827 & 2,773 & 16 \\
\hline
\end{tabular}

DE: desviación estándar / DE: standard deviation.

insectos han demostrado que se requiere una dosis letal media mayor para estos productos en comparación con el flupyradifurone, mostrando diferencias significativas (Smith et al., 2016).

La combinación de un insecticida con un organismo de control biológico, como los hongos entomopatógenos ha sido empleada para el control de plagas agrícolas, debido a que el insecticida debilita al insecto, le provoca estrés y altera su comportamiento, lo que es aprovechado por el organismo entomopatógeno para mejorar su efecto de control en la plaga. Esta combinación también permite reducir la carga química, el impacto al ambiente y acelerar el proceso de muerte del insecto para el control de las plagas, a diferencia del tiempo que requiere un entomopatógeno solo para matar al huésped (Boucias et al., 1996; Quintela y McCoy, 1998).

Otros estudios han sido realizados para evaluar la combinación del hongo entomopatógeno Methrhizium anisopliae con el insecticida imidacloprid para el control de Aedes aegypti, los resultados demostraron que esta combinación fue el mejor tratamiento para control de A. aegypti (Paula et al., 2011).

Estudios similares al presente, donde se evaluó el efecto del imidacloprid y el fipronil con los hongos entomopatógenos Beauveria bassiana y Metarhizium anisopliae sobre el comportamiento de las termitas Heterotermes tenuis, demostraron que la combinación del imidacloprid más B. bassiana trabaja de forma sinérgica y por ello, se obtiene un mejor control sobre las poblaciones de insectos plaga. Es posible que el fipronil no diera los mismos resultados por el modo de acción de su ingrediente activo sobre el insecto. El imidacloprid es un insecticida neonicotinoide, sistémico, que actúa como una neurotoxina ligada a los receptores nicotínicos de la acetilcolina, donde la capacidad de estresar al insecto favorece la acción del hongo actuando de forma sinérgica (Moino y Alves, 1998).

El modo de acción de flupyradifurone consiste en actuar como un agonista del receptor nicotínico acetilcolina del insecto (nAChR), de esta forma imita al neurotransmisor de la acetilcolina, lo cual provoca que este no puede ser activado por la enzima respectiva, en su forma natural, y causa excitación del nervio de la célula, la persistencia de este efecto provoca una alteración el en nervio del insecto y, posteriormente, su colapso (Nauen et al., 2015). La combinación de flupyradifurone con B. bassiana podría ser beneficioso cuando el sistema nervioso del insecto empieza a afectarse, permitiéndole al hongo actuar y provocar su muerte por ambas vías.

El empleo de este agroquímico ha sido reportado seguro para las abejas, como lo indican Hesselbach y Scheiner (2018) en su estudio, donde demostraron que solo en dosis altas podría afectar las abejas, sin embargo, en un programa de MIP se busca que las aplicaciones no sean en horas donde las abejas están en una alta actividad para su protección. 
Otro estudio que buscó la combinación de flupyradifurone con otro elemento para el control de plagas fue realizado por Kumar et al. (2017), donde combinaron flupyradifurone con Amblyseius swirskii para el control de mosca blanca; los resultados estadísticos indicaron que el mejor tratamiento fue la combinación de flupyradifurone + Amblyseius swirskii, al ser comparado con los tratamientos de flupyradifurone y Amblyseius swirskii aplicados individualmente. También demostraron que el flupyradifurone no afectó las poblaciones del ácaro Amblyseius swirskii.

El mejor rendimiento de las parcelas evaluadas lo presentaron los tratamientos de flupyradifurone $\left(11 \mathrm{ha}^{-1}\right)+B$. bassiana y flupyradifurone $\left(0,751 \mathrm{ha}^{-1}\right)+B$. bassiana (Cuadro 3). En el rendimiento del cultivo se tomó en cuenta el porcentaje de rechazo provocado principalmente por virosis en el vaina del ejote francés. En estudios realizados con el plaguicida flupyradifurone en comparación con tiametoxam, se determinó que el primero además de tener mejor control sobre mosca blanca, también ejerció una mejor supresión del virus TYCV, con un $85 \%$, mientras que el tiametoxam controló solo un $25 \%$ (Roditakis et al., 2017).

La distribución de adultos de la mosca blanca en la planta del ejote francés presentó diferencias significativas en los tres estratos de la planta (alto, medio y bajo) (Cuadro 4); la mayor población de la mosca blanca se ubicó en los estratos alto y medio, esto pudo deberse a que, estos correponden a hojas más jóvenes, donde las moscas pueden mostrar preferencia para su alimentación. En un estudio similar en cuanto a la distribución de ninfas y adultos de $T$. vaporariorum en el cultivo del tomate, estos mostraron preferencias significativas en los foliolos sétimo y octavo, las cuales son hojas más jóvenes (Bernal et al., 2008).

En la comparación de las poblaciones de mosca blanca por estrato en la época seca y época lluviosa, se contabilizó mayor población de mosca en el estrato alto en época lluviosa, esto puedo deberse a que esa época pudo perderse la efectividad de los tratamientos por las lluvias, por lo que, el mejor control de la población se obtuvo en la época seca. Otro estudio realizado en la distribución de las ninfas, en el cultivo del tomate (Bernal et al., 2008), mostró la preferencia de las ninfas por el tercio inferior y parte media de la planta del tomate, presentando estas áreas hojas jóvenes.

Trialeurodes vaporariorum fue la especie más abundante en el cultivo del ejote francés, tomando en cuenta que predominó en un $95 \%$ su población, mientras que $B$. tabaci con un $5 \%$, esto podría indicar que la efectividad de los tratamientos evaluados fue para T. vaporariorum, recomendando hacer la misma evaluación donde las poblaciones de B. tabaci sean representativas.

\section{Conclusiones}

El mejor tratamientos para el control de la mosca blanca Trialeurodes vaporariorum (Westwood)], fue flupyradifurone $\left(0,751 \mathrm{ha}^{-1}\right)+B$. bassiana $\left(4,541 \mathrm{ha}^{-1}\right)$. El cual también demostró la protección del cultivo obteniendo mejores rendimientos y mejor calidad de la vaina cosechada. Es el tratamiento que emplea la dosis mínima recomendada por el fabricante, lo que permite una menor carga química en el cultivo.

Según los resultados obtenidos, en el manejo de las poblaciones de mosca blanca es importante tomar en cuenta la distribución de la población, las cuales se encontraron principalmente en los estratos alto y medio de la planta de ejote francés.

La información obtenida en la distribución de la población de la mosca blanca en el cultivo del ejote francés también permitirá dirigir las aplicaciones de plaguicidas para su control principalmente en los estratos medio y alto. Esto garantizará mejores resultados en su control, principalmente con plaguicidas de acción de contacto.

Conocer la especie dominante del complejo de mosca blanca en un cultivo es importante ya que esta información permite tomar decisiones para establecer planes de manejo integrado, y en el caso de los controladores biológicos, poder hacer una correcta elección del enemigo natural específico, que permita un mejor control poblacional. 


\section{Agradecimientos}

Esta investigación fue cofinanciada por la Dirección General de Investigación, de la Universidad de San Carlos de Guatemala (Digi-Usac) en el año 2018, proyecto presupuestado con la partida 4.8.63.4.07. Se agradece al Lic. Federico Nave por su valiosa colaboración en el análisis estadístico de la presente investigación.

\section{Literatura citada}

AGEXPORT (Asociación Guatemalteca de Exportadores). 2016. Sector de arvejas y vegetales potencializará su promoción en destinos internacionales. AGEXPORT, GTM. http://agexporthoy.export.com.gt/sectores-de-exportacion/sector-agricola/ comite-de-arveja-y-vegetales-busca-incrementar-la-promocion-en-los-mercados-internacionales-y-mejorar-el-acceso/ (consultado 18 may. 2018).

AGEXPORT (Asociación Guatemalteca de Exportadores). 2018. Ejote francés. AGEXPORT, GTM. http://portal.export.com.gt/ portal/clientes/tecnicas_nacionales/ejote\%20frances.pdf (consultado 7 ene. 2018).

AMSA (Autoridad para el Manejo Sustentable de la Cuenca de Lago de Amatitlán). 2005. Monografía del Municipio de Parramos. Editorial José Pineda Ibarra, GTM.

Bernal, L., L. Pesca, D. Rodríguez, F. Cantor, y J. Cure. 2008. Plan de muestreo directo para Trialeurodes vaporariorum (Westwood) (Hemiptera: Aleyrodidae) en cultivos comerciales de tomate. Agron. Colomb. 26:266-276.

Boucias, D.G., C. Stokes, G. Storey, and J.C. Pendland. 1996. The effects of Imidacloprid on the termite Reticulitermes flavipes and its interaction with the mycopathogen Beauveria bassiana. Pflanzenschutz-Nachr. Bayer 49:103-144.

Bueno, J.M., C. Cardona, y P. Chacón. 2005. Fenología, distribución espacial y desarrollo de métodos de muestreo para Trialeurodes vaporariorum (Westwood) (Hemiptera: Aleyrodidade) en habichuela y fríjol (Phaseolus vulgaris L.). Rev. Colomb. Entomol. 31:161-170.

Caballero, R. 1992. Whiteflies (Homopetera: Aleyrodidae) from Central America and Colombia field keys including slidemounted pupal and field keys for identification, field characteristics, hosts, distribution, natural enemies and economic importance. MSc. Diss., Kansas State University, KS, USA.

Cámara del Agro, y Agrequima. 2015. El AGRO es vital para la economía del país: Elementos de propuesta de política agrícola para Guatemala. Agrequima, GTM. https://agrequima.com.gt/site/wp-content/uploads/2017/05/Elementos-de-PropuestaPolitica-para-Guatemala.pdf (consultado 27 abr. 2018).

Campuzano-Martínez, A., J.C. Rodríguez-Maciel, A. Lagunes-Tejeda, C. Llanderal-Cázares, A.T. Teran-Vargas, y J. Vera-Graciano. 2010. Aptitud biológica de poblaciones de Bemisia tabaci (Gennadius) biotipo b (Hemiptera: Aleyrodidae) con diferente susceptibilidad al insecticida thiametoxam. Neotrop. Entomol. 39:430-435. doi:10.1590/ S1519-566X2010000300018

Carapia, V., y A. Castillo. 2013. Estudio comparativo sobre la morfología de Trialeurodes vaporarorium (Westwood) y Bemisia tabaci (Gennadius) (Hemiptera: Aleyrodidae). Rev. Colomb. Entomol. 35:177-184.

Contreras, B.H. 2014. Evaluación cualitativa de la cadena de valor de vegetales de exportación en Guatemala. PRONACOM, GTM. https://www.pronacom.gt/website/biblioteca/biblioteca_10._vegetales.pdf (consultado 31 may. 2019).

Cuellar, M.E., y F.J. Morales. 2006. La mosca blanca Bemisia tabaci (Gennadius) como plaga y vectora de virus en frijol común (Phaseolus vulgaris L.). Rev. Colomb. Entomol. 32:1-9. 
EC (European Commission). 2017. EU pesticides database. EC. http://ec.europa.eu/food/plant/pesticides/eu-pesticidesdatabase/public/?event=product.resultat\&language=EN\&selectedID=179 (accessed Jun. 5, 2019).

Espinel, C., L. Torres, E. Grijalba, L. Villamizar, y A. Cortes. 2008. Preformulados para control de la mosca blanca Bemisia tabaci (Hemiptera: Aleyrodidae) en condiciones de laboratorio. Rev. Colomb. Entomol. 34(1):22-27.

Hesselbach, H., and S. Scheiner. 2018. Effects of the novel pesticide flupyradifurone (Sivanto) on honeybee taste and cognition. Sci. Rep. 8:4954. doi:10.1038/s41598-018-23200-0

INE (Instituto Nacional de Estadísticas). 2013. Caracterización departamental Chimaltenango 2013. INE, GTM. https://www. ine.gob.gt/sistema/uploads/2015/07/20/uYeKswXf9BHOJHZZZ7f7eR8CaMWVXnzg.pdf (consultado 23 jun. 2018).

Jones, D.R. 2003. Plant viruses transmitted by whiteflies. Eur. J. Plant Pathol. 109:195-219. doi:10.1023/A:1022846630513

Kandil, M.A., A.Y. Saleh, W.H. El-Dieb, and S.F. Farghaly. 2008. Resistance mechanisms of whitefly Bemisia tabaci (Homoptera: Aleyrodidae) to thiamethoxam and propenofos. Asian J. Biol. Sci. 1:33-38. doi:10.3923/ajbs.2008.33.38

Kumar, V., G. Kakkar, C.L. McKenzie, and L.S. Osborne. 2017. Efficacy of foliar application of Flupyradifurone on Bemisia tabaci (MED Whitefly) and Amblyseius swirskii. Arthropod Manag. Tests 42(1):tsx132. doi:10.1093/amt/tsx132

Macías-Flores, A., C. Santillán-Ortega, A. Robles-Bermudez, M. Ortíz-Cantón, and O. Cambero-Campos. 2013. Selected events of resistance to insecticides in whiteflies (Hemiptera: Aleyrodidae) in the world. Rev. BioCienc. 2(2):4-16.

Moino, A., e S.B. Alves. 1998. Efeito de Imidacloprid e Fipronil sobre Beauveria bassiana (Bals.) Vuill.e Metarhizium anisopliae (Metsch.) Sorok. e no comportamento de limpeza de Heterotermes tenuis (Hagen). Anis Soc. Entomol. Bras. 27(4):611-619. doi:10.1590/S0301-80591998000400014

Morales, F. 2006. History and current distribution of begomoviruses in Latin America. Plant Virus Epidemiol. 67:127-162. doi:10.1016/S0065-3527(06)67004-8

Morales, F., and P. Anderson. 2001. The emergence and dissemination of whitefly-transmitted geminiviruses in Latin America. Arch. Virol. 146:415-441. doi:10.1007/s007050170153

Nauen, R., P. Jeschke, R. Elten, M. Beck, U. Ebbinghaus-Kintscher, W. Thielert, K. Wölfel, M. Haas, K. Kunz, and G. Raupach. 2015. Flupyradifurone: a brief profile of a new butenolide insecticide. Pest Manag. Sci. 71:850-862. doi:10.1002/ps.3932.

Naveen, N.C., R. Chaubey, D. Kumar, K.B. Rebijith, R. Rajagopal., B. Subrahmanyam, and S. Subramanian. 2017. Insecticide resistance status in the whitefly, Bemisia tabaci genetic groups Asia-I, Asia-II-1 and Asia-II-7 on the Indian subcontinent. Sci. Rep. 7:40634. doi:10.1038/srep40634

Ouma, B., J. Muthomi, J. Nderitu, and F. Toroitich. 2014. Management of thrips in french bean by integrating biological and synthetic pesticides in conventional spray regimes. JRA 2(2):27-37. doi:10.12966/jra.06.02.2014

Paula, A.R., A.T. Carolino, C.O. Paula, and R.I. Samuels. 2011. The combination of the entomopathogenic fungus Metarhizium anisopliae with the insecticide Imidacloprid increases virulence against the dengue vector Aedes aegypti (Diptera: Culicidae). Parasit. Vectors 4:8. doi:10.1186/1756-3305-4-8

Quintela, E.D., and C.W. McCoy. 1998. Conidial attachment of Metarhizium anisopliae and Beauveria bassiana to the larval cuticle of Diaprepes abbreviatus (Coleoptera: curculionidae) treated with imidacloprid. J. Invertebr. Pathol. 72:220-230. doi:10.1006/jipa.1998.4791

Roditakis, E., M. Stavrakaki, M. Grispou, A. Achimastou, X. Van-Waetermeulen, R. Nauen, and A. Tsagkarakou. 2017. Flupyradifurone effectively manages whitefly Bemisia tabaci MED (Hemiptera: Aleyrodidae) and tomato yellow leaf curl virus (TYLCV) in tomato. Pest Manag. Sci. 73:1574-1584. doi:10.1002/ps.4577 
Syngenta. 2019. Serengueti Syngenta. Semilla híbrida de habichuela. Syngenta, GTM. https://www.syngenta.com.gt/serengeti (consultado 21 feb. 2019).

Salas, J., and O. Mendoza. 1995. Biology of the sweetpotato whitefly (Homoptera: Aleyrodidae) on tomato. FL Entomol. 78:154-160. doi:10.2307/3495680

Smith, H.A., and M.C. Giurcanu. 2013. Residual effects of new insecticides on egg and nymph densities of Bemisia tabaci (Hemiptera: Aleyrodidae). FL Entomol. 96:504-511. doi:10.1653/024.096.0216

Smith, H.A., C.A. Nagle, C.A. MacVean, and C.L. McKenzie. 2016. Susceptibility of Bemisia tabaci MEAM1 (Hemiptera: Aleyrodidae) to imidacloprid, thiamethoxam, dinotefuran and flupyradifurone in south Florida. Insects 7(4):E57. doi:10.3390/I.2016-7040057

U.S. Government Publishing Office. 2019. Electronic code of federal regulations. U.S. Government Publishing Office, USA. https://www.ecfr.gov/cgi-bin/text-idx?SID=b086a3f2b21c697c598b175abe3ad427\&mc=true \&node=se40.26.180_1679 \&rgn=div8 (accessed May. 31, 2019).

Villar-Sánchez, B., E. López-Salinas, y J. Acosta-Gallegos. 2003. Selección de genotipos de frijol por rendimiento y resistencia al mosaico dorado y suelos ácidos. Rev. Fitotec. Mex. 26(2):109-114. 